\title{
Relative Properties and Relative Potency of Various Hydrazide Compounds That Inhibit Growth of Mycobacterium tuberculosis
}

\author{
Ronald Bartzatt ${ }^{1}$ \\ ${ }^{1}$ (University of Nebraska, Durham Science Center, 6001 Dodge Street, Omaha Nebraska 68182 USA)
}

\begin{abstract}
The molecular properties of compounds expressing growth inhibition of Mycobacterium tuberculosis are determined and examined for underlying relationships by pattern recognition methods. Pattern recognition methods such as hierarchical cluster analysis will show relationships between compounds. Descriptive statistical analysis of molecular properties reveal commonality among bacteriostatic compounds. Outliers within any category of descriptor is identified by Grubb's test. Potency of growth inhibition measured as MIC (minimum inhibitory concentration) after in vitro evaluation will be compared and analyzed. Structure moieties and their effect on activity is identified. All compounds analyzed showed substantial growth inhibition of Mycobacterium tuberculosis, having MIC values ranging from $16.7 \mu \mathrm{grams} / \mathrm{milliliter}$ to $65.9 \mu \mathrm{grams} / \mathrm{milliliter}$ (isoniazid MIC at $14.7 \mu$ grams/milliliter). Ranges in values of Log $P$, number of atoms, and molecular weight were, respectively: -1.89 to $1.10,11$ to 25 (isoniazid at 10), and 150.18 grams $/$ mole to $345.38 \mathrm{grams} / \mathrm{mole}$ (isoniazid at 137.14). All eight compounds and isoniazid showed favorable bioactivity within six categories of measureable bioactivity scores. The molecular properties of eight compounds and isoniazid varied, however, remained favorable in drug-likeness with zero violations of the Rule of 5. Interrelationships of the descriptors were revealed by statistical analysis.
\end{abstract}

Keywords: Mycobacterium tuberculosis, tuberculostats, hydrazide, antimicrobial

\section{Introduction}

Up to 3 million deaths due to Mycobacterium tuberculosis are thought to occur each year, along with up to 8 million new cases of infection [1]. With nearly one-third of the world's population believed to be infected with Mycobacterium tuberculosis, several outbreaks of multidrug resistant tuberculosis were observed during the 1990's in Europe and the United States [1]. Multidrug-resistant tuberculosis (MDR-TB) is defined to be bacilli resistant to at least two members of the first-line tuberculostats, notably isoniazid and rifampin [2]. In 2008, up to 510000 cases of MDR-TB or 3.6\% of all incidents, were observed across the globe [2]. Treatment of MDR-TB is more difficult, less effective, and even more than 100 times costlier than drug susceptible tuberculosis [2]. Isoniazid is a crucial member of the first-line combination therapy regimen of tuberculosis treatment [2].The description of extensively drug-resistant tuberculosis (XDR-TB) are those incidents of infection resistant to isoniazid, rifampin, any fluoroquinolone, and at least one second-line drug (amikacin, kanamycin, capreomycin) [3]. XDR-TB is more difficult to treat and has been identified in 46 countries by 2008 [3]. In 2006 as many as 200000 individuals, co-infected with HIV, died of complications with tuberculosis [3].

There is an urgent necessity to improve clinical treatment of tuberculosis, by improving the application of existing drugs and/or development of new agents [4]. The definition of first-line treatment agents includes rifampin, isoniazid, pyrazinamide, and ethambutol, which are taken over a period of at least six months to acquire high cure rates of more than 95\% [4]. Adverse effects to anti-tuberculosis drugs are commonly seen and contribute to problem of patient adherence to therapy [4]. Second-line agents are even more toxic, less effective, and require even longer use than first-line agents [4]. MDR-TB treatment requires the application of the more expensive second-line agents for at least 18 months $[\mathbf{5 , 6 ]}$. A rise of the number of incidents of retreatment results in the increase of drug-resistance in any region [7]. By 2009, it was estimated that $11.1 \%$ of new cases of tuberculosis demonstrated resistance to any drug with that figure rising to $25.1 \%$ in cases of previous treatment [8]. The clinical outcome for patients harboring XDR-TB is even worse than that for MDR-TB [9], is spread through the air like other forms of tuberculosis [10], and shows a much higher mortality rate that MDRTB [11]. Tuberculosis infection is a health threat having significant morbidity and mortality, with up to 1.5 million deaths annually [12]. Drug-resistant strains of bacterial tuberculosis have increased, as well as viral HIV co-infection with tuberculosis [12]. Up to one-third of the world's population is thought to be infected with Mycobacterium tuberculosis $[\mathbf{1 3}, \mathbf{1 4}]$. Notably, each year up to 8.8 million patients are newly diagnosed with an active infection of tuberculosis [14]. To complicate and make more difficult the clinical treatment of tuberculosis, the current first-line drugs for treatment (isoniazid, rifampin, pyrazinamide, ethambutol) must be taken for up to 6 months to achieve high level of cure rates (>95\%) [14]. Therefore, the study and development of new drugs is vital for the clinical treatment of tuberculosis. 


\section{Properties and Molecular Modeling}

\section{Materials And Methods}

Various numerical values of molecular properties (descriptors) of compound structures were accomplished by utilizing Molinspiration (Molinspiration Cheminformatics, Nova ulica 61, SK-900 26 Slovensky Grob, Slovak Republic). Determination of molecular structure components was accomplished by utilizing ACD ChemSketch Modeling v. 12.01 (Advanced Chemistry Development, 110 Yonge Street, Toronto Ontario, M5C 1T4 Canada, http://www.molinspiration.com/services/search.html). Determination of druglikeness scores for kinase inhibitor, protease inhibitor, and enzyme inhibitor, etc., was determined by Molinspiration Cheminformatics (http://www.molinspiration.com/cgi-bin/properties).

\section{Pattern Recognition and Multivariate Analysis}

Various pattern recognition techniques to identify underlying associations and patterns within the numerical properties utilizing hierarchical cluster analysis accomplished by KyPlot v. 2.0 Beta 15 (copyright Koichi Yoshioka 1997-2001). Analysis of one-way ANOVA and 95\% ellipses was performed by PAST v. 2.06 (copyright Oyvind Hammer, D.A.T. Harper 1999-2008). Path analysis is accomplished by OpenStat (copyright William Miller, September 11, 2008).

\section{Various Statistical Analysis}

Other statistical analysis of numerical data that includes Pearson $r$ correlation and various descriptive statistics was accomplished by Microsoft EXCEL v. 14.0.6112.5000 (EXCEL Professional plus 2010). Multiple regression analysis of molecular property values was accomplished utilizing GraphPad Instat version 3.00 (GraphPad Software, Inc., San Diego California USA; www.graphpad.com). Identification of numerical outliers was accomplished by Grubb's test (extreme studentized deviate), with the t-test to compare two means was done by (http://www.graphpad.com/quickcalcs/).

\section{Structure and Properties}

\section{Results And Discussion}

The development of new tuberculostats are of great importance on account the appearance of multidrug resistant, extensively drug resistant, and totally drug resistant tuberculosis [2,3]. In this investigation, a group of novel hydrazide compounds have been compared to the first-line drug isoniazid for effectiveness of Mycobacterium tuberculosis inhibition. The effectiveness of inhibition is measured by in vitro method in which various compounds are added in known increasing concentration $[15,16]$.Molecular structures of all compounds tested in vitro and evaluated in this study $[\mathbf{1 5}, \mathbf{1 6}]$, are presented in Figure 1 for comparison to the first-line drug isoniazid (compound 9) and other hydrazide compounds. Notably, a characteristic of all the compounds shown, is the hydrazide group $(-\mathrm{C}(=\mathrm{O})-\mathrm{NHNH} 2)$ that is a substituent for all molecular structures. All compounds presented in Figure 1 have one aromatic ring (containing a conjugated planar ring system), with various substituents covalently bonded onto the aromatic ring (see Figure 1).

In addition to the hydrazide group, the compounds 1 to 9 have various substituents. Compound 1 has a methyl group substituent (-CH3) in meta position to the hydrazide group. Compound 2 with acetate ($\mathrm{OC}(=\mathrm{O}) \mathrm{CH} 3)$ group in meta position to hydrazide. Compound 3 has a chloromethyl group $(-\mathrm{CH} 2 \mathrm{Cl})$ to hydrazide. Compound 4 has a single nitro group $(-\mathrm{NO} 2)$ in para position relative to the hydrazide. Compound 5 has a bromine atom in ortho position relative to hydrazide. Compound 6 has a bromine atom in the meta position to hydrazide. Compound 7 has a bromine atom in the para position relative to the hydrazide. Compound 8 is ciprofloxacin having a hydrazide group in place of the original carboxyl group $(-\mathrm{C}(=\mathrm{O}) \mathrm{OH})$. Compound 9 is the first-line tuberculostat isoniazid.

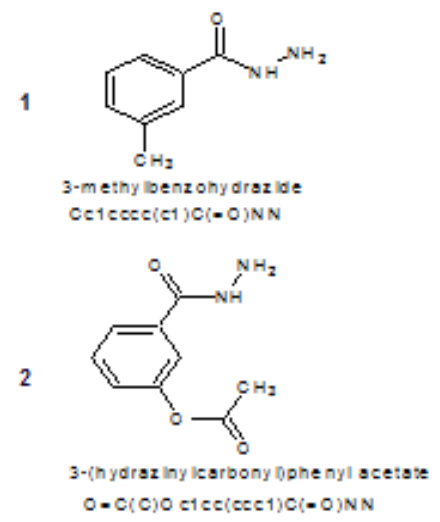

6

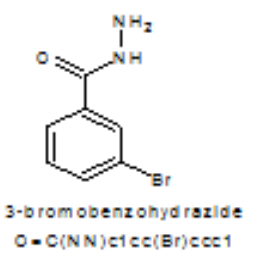

7

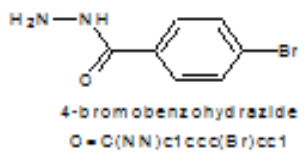




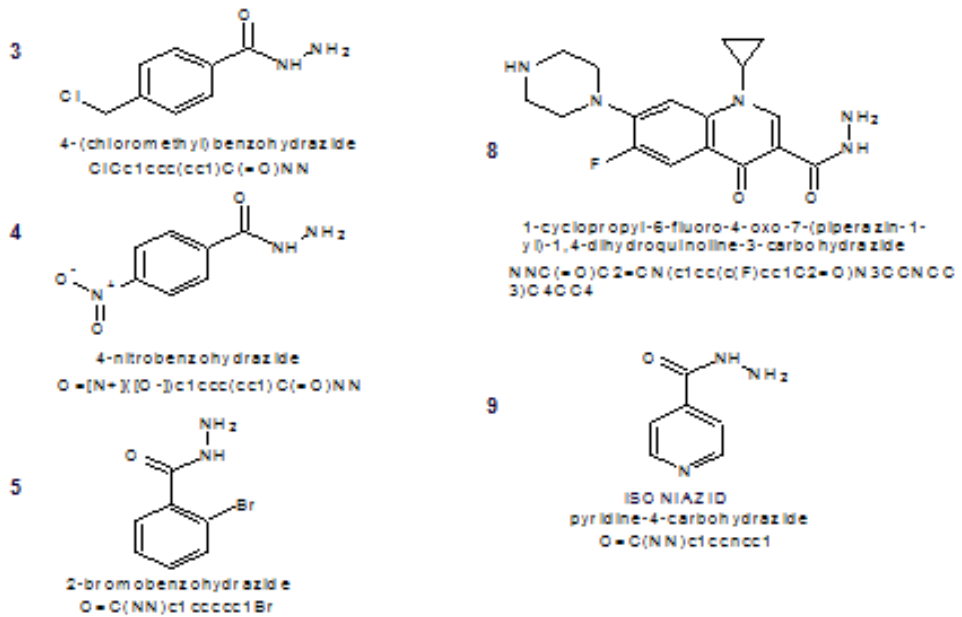

Figure 1 Molecular structures of hydrazide compounds that inhibit Mycobacterium tuberculosis with IUPAC designation and SMILES notation.

The effects of various substituent groups covalently bound to the aromatic ring within these compounds influences the molecular properties (Log P, polar surface area, molecular weight, etc) and thereby the bioavailability (the fraction of an administered dose of unchanged drug that reaches the systemic circulation) of a potential drug $[17, \mathbf{1 8}]$. Drug-likeness is a qualitative concept used in drug design as a measure of how drug-like a substance is with respect to factors like bioavailability [17].

Physical properties of the drug affect the bioavailability of any drug [17]. Various molecular properties have been shown to be highly useful for evaluating the drug-likeness of a potential drug and various screening parameters assist in identifying compounds for advanced development $[\mathbf{1 7}, \mathbf{1 8}, \mathbf{1 9}]$. The Rule of 5 is a screening test to determine if a chemical compound with a certain biological activity has properties that would make it orally active in humans $[\mathbf{1 7}, \mathbf{1 8}]$. This rule states that, in general, an orally active drug has no more than one violation of the following criteria [17, 18]: 1) No more than 5 hydrogen bond donors (total number of nitrogenhydrogen and oxygen-hydrogen bonds); 2) No more than 10 hydrogen bond acceptors (all nitrogen and oxygen atoms); 3) A molecular mass less than 500 grams/mole; and 5) An octanol-water partition coefficient $\log P$ not greater than 5. Another screening test for favorable drug-likeness is condensed to meet only two rules [19]: 1) Ten or fewer rotatable bonds, and 2) Polar surface area less than 140 Angstroms $^{2}$. All compounds 1 to 9 show zero violations of the Rule of 5 (see Table 1), indicating favorable drug-likeness and bioavailability [17].The molecular properties of all nine compounds are listed in Table 1 and show the diverse effects of various substituents on molecular properties and consequently the drug-likeness of these compounds.

Table 1 Molecular Properties of Compounds

\begin{tabular}{|c|c|c|c|c|c|c|c|c|c|}
\hline \multirow[b]{2}{*}{ Property } & \multicolumn{9}{|c|}{ COMPOUNDS } \\
\hline & 1 & 2 & 3 & 4 & 5 & 6 & 7 & 8 & $\begin{array}{l}9 \\
\text { isoniazid }\end{array}$ \\
\hline $\log P$ & 0.74 & -0.15 & 0.90 & 0.28 & 1.08 & 1.10 & 1.10 & -1.89 & -0.97 \\
\hline $\begin{array}{l}\text { Polar Surface area } \\
\left(\text { Angstroms }{ }^{2}\right)\end{array}$ & 55.12 & 81.43 & 55.12 & 100.94 & 55.12 & 55.12 & 55.12 & 92.39 & 68.01 \\
\hline Number of atoms & 11 & 14 & 12 & 13 & 11 & 11 & 11 & 25 & 10 \\
\hline Molecular Weight & 150.18 & 194.19 & 184.63 & 181.15 & 215.05 & 215.05 & 215.05 & 345.38 & 137.14 \\
\hline $\begin{array}{l}\text { Number of oxygen } \\
\& \text { nitrogen atoms }\end{array}$ & 3 & 5 & 3 & 6 & 3 & 3 & 3 & 7 & 4 \\
\hline $\begin{array}{l}\text { Number of }-\mathrm{OH} \\
\text { and }-\mathrm{NH}_{\mathrm{n}}\end{array}$ & 3 & 3 & 3 & 3 & 3 & 3 & 3 & 4 & 3 \\
\hline $\begin{array}{l}\text { Violations of Rule } \\
\text { of Five }\end{array}$ & 0 & 0 & 0 & 0 & 0 & 0 & 0 & 0 & 0 \\
\hline $\begin{array}{ll}\text { Number } & \text { of } \\
\text { Rotatable Bonds }\end{array}$ & 1 & 3 & 2 & 2 & 1 & 1 & 1 & 3 & 1 \\
\hline $\begin{array}{l}\text { Molecular Volume } \\
\left(\text { Angstroms }{ }^{3}\right)\end{array}$ & 143.28 & 171.25 & 157.06 & 150.05 & 144.60 & 144.60 & 144.60 & 301.13 & 122.56 \\
\hline $\begin{array}{l}\text { Minimum } \\
\text { Inhibitory } \\
\text { Concentration } \\
\text { ( } \mu \text { gram/milliliter) }\end{array}$ & 35.0 & 35.0 & 35.0 & 35.0 & 65.9 & 29.5 & 21.5 & 16.7 & 14.7 \\
\hline $\log B B$ & -0.563 & -1.01 & -0.541 & -1.31 & -0.513 & -0.485 & -0.485 & -1.07 & -0.973 \\
\hline BB & 0.273 & 0.0977 & 0.288 & 0.0487 & 0.307 & 0.327 & 0.327 & 0.0857 & 0.106 \\
\hline
\end{tabular}


Oral administration is the method of choice to insert a drug in humans [20]. The portion of drug administered that is absorbed intact depends on various processes as well as membrane permeability [20]. With membrane permeation as a common requirement for oral bioavailability, other estimated interactions of physicochemical descriptors and intestinal absorption can be used for studies of drug structure-bioavailability [20]. Lipophilicity is defined as the affinity of a drug molecule for a lipophilic environment and is a key property in the transport processes of drugs including intestinal absorption, membrane permeability, protein binding, and distribution among tissues [20]. Aqueous solubility is a vital property that will influence drug release and its permeability through biological membranes and absorption in humans [20]. Consequently, it is important to estimate water solubility from molecular structure and physicochemical descriptors [20]. Tuberculosis can enter the central nervous system with high morbidity and mortality [15]. Previous studies have determined means to estimate drug penetration into the central nervous system (as Log Cbrain/Cblood or Log $\mathrm{BB})$ and that equation $\{1\}$ can be applied effectively utilizing polar surface area (PSA) and Log P [21]:

$$
\log \mathrm{BB}=-0.0148(\mathrm{PSA})+0.152(\log \mathrm{P})+0.139
$$

Values of $\log$ BB and corresponding BB ratios for compounds 1 to 9 are shown in Table 1.Values of BB (Cbrain/Cblood) range from 0.0487 (compound 4) to 0.327 (compounds 7 and 8). Although values of BB vary for these compounds, the Grubb's test (extreme studentized deviate) [22, 23] shows no outliers in these numerical values of $\mathrm{BB}$, inclusive of isoniazid. Notably the penetration of the central nervous system (CNS) by compounds $1,3,5,6$, and 7 is greater (by values of $\mathrm{BB}$ ) than that for first-line tuberculostat isoniazid. Clearly, the judicious choice of structure design can improve the vital ability of CNS penetration for clinical treatment of CNS tuberculosis.

Grubb's test for outliers [22, 23] indicates no outliers within values of $\log P$, polar surface area, number of oxygen \& nitrogen atoms, number of rotatable bonds, and Log BB values (see Table 1).

Comparison of all properties (Table 1) for all nine compounds, even though the numerical values vary, Pearson $r$ correlation of compound to other compounds indicates a very strong positive relationship $r>0.9700$. One-way ANOVA analysis of all molecular properties for all compounds indicate that the means of the numerical values for each compound are essentially equal $(P=.98)$ [22, 23].Biological activity, used in drug development, describes the beneficial or the adverse effects of a drug on living tissue and this activity is expressed through the agents pharmacophore. Biological activity is a crucial expression of the agent's possible uses in any medical application. Compounds may show some adverse and toxic effects which may prevent their clinical application $[18,19,20]$.

Table 2 shows the distribution of bioactivity scores of all nine compounds for the six most important categories of drugs (GPCR ligands, ion channel modulators, kinase inhibitors, nuclear receptor ligands, protease inhibitors, other enzyme inhibitors). The range for average drug-like molecules for each category is provided for each category in parenthesis (see Table 2). Note that all nine compounds fall within the drug-like optimal range for each of the six categories of drugs. Although the numerical values of bioactivity for this group of agents vary and are diverse, the Pearson $r$ correlation for compound to compound is greater than 0.8900 , indicating a very strong positive relationship [22, 23]. However, the median of numerical values for each compound are not equal by Kruskal-Wallis test $(P=.002)$. In addition, the means of numerical values for each compound are not equal to that of other compounds, by one-way ANOVA $(P<0.001)$. Consequently, for these hydrazide compounds, including isoniazid, the bioactivity scores are favorable, diverse, and vary but not identical from compound to compound.

Table 2 Bioactivity of Compounds Compared to Drug Categories

\begin{tabular}{|l|l|l|l|l|l|l|l|l|l|}
\hline \multirow{2}{*}{$\begin{array}{l}\text { Category } \\
\text { (optimum range) }\end{array}$} & $\mathbf{1}$ & $\mathbf{2}$ & $\mathbf{3}$ & $\mathbf{4}$ & $\mathbf{5}$ & $\mathbf{6}$ & $\mathbf{7}$ & $\mathbf{8}$ & $\begin{array}{l}\mathbf{9} \\
\text { isoniazid }\end{array}$ \\
\cline { 2 - 9 } $\begin{array}{l}\text { GPCR ligand } \\
(-1.5 \text { to 0.50) }\end{array}$ & -1.40 & -1.04 & -1.41 & -1.31 & -1.65 & -1.58 & -1.58 & -0.10 & -1.39 \\
\hline $\begin{array}{l}\text { Ion channel } \\
\text { modulator } \\
(-2.0 \text { to 0.50) }\end{array}$ & -1.59 & -1.13 & -1.43 & -1.22 & -1.68 & -1.61 & -1.61 & -0.61 & -1.45 \\
\hline $\begin{array}{l}\text { Kinase inhibitor } \\
(-2.0 \text { to 0.50) }\end{array}$ & -1.29 & -1.02 & -1.49 & -1.16 & -1.44 & -1.37 & -1.37 & -0.02 & -1.05 \\
\hline $\begin{array}{l}\text { Nuclear receptor } \\
\text { ligand } \\
(-2.0 \text { to 0.50) }\end{array}$ & -2.04 & -1.24 & -2.23 & -1.76 & -2.27 & -2.31 & -2.31 & -0.71 & -2.33 \\
\hline $\begin{array}{l}\text { Protease inhibitor } \\
(-2.0 \text { to 0.50) }\end{array}$ & -1.25 & -0.83 & -0.84 & -1.15 & -1.54 & -1.45 & -1.45 & -0.27 & -1.23 \\
\hline $\begin{array}{l}\text { Enzyme inhibitors } \\
(-1.5 \text { to 0.50) }\end{array}$ & -0.84 & -0.48 & -0.66 & -0.73 & -0.98 & -0.90 & -0.90 & 0.12 & -0.66 \\
\hline
\end{tabular}




\section{Pattern Recognition and Comparative Potency}

Pattern recognition focuses on the recognition of patterns and regularities in data, identifying underlying relationships [22, 23]. Cluster analysis or clustering is technique of pattern recognition, which groups (drugs) a set of objects in such a way that objects in the same group (or cluster) are more most similar to each other than to those found in other groups (clusters) $[\mathbf{2 2}, \mathbf{2 3}]$. Hierarchical cluster analysis is based on objects (drugs) being more related to nearby objects than to objects farther away. Single-linkage clustering is based on grouping clusters combining two clusters that contain the closest pair of elements or when the two clusters whose two closest members have the smallest distance [23].

Cluster analysis applied in drug development can identify, utilizing molecular properties, all agents that are most similar. Applied in this study, based on molecular properties (see Table 1), hierarchical cluster analysis identified which compounds have highest level of similarity (see Figure 2). The outcome presented in Figure 2 as a vertical dendrogram, beginning at super node A, under node B compounds 4 and 9 (isoniazid) are determined to be most similar. Under node $\mathrm{C}$, compounds $1,7,8,5$, and 6 are determined to have the highest similarity. However, under node $\mathrm{C}$, compound 2 is determined to be distinct from $1,7,8,5,6$, and 3 . Therefore, compound 4 may be most similar to isoniazid in clinical treatment of tuberculosis.

\section{Dendrogram}

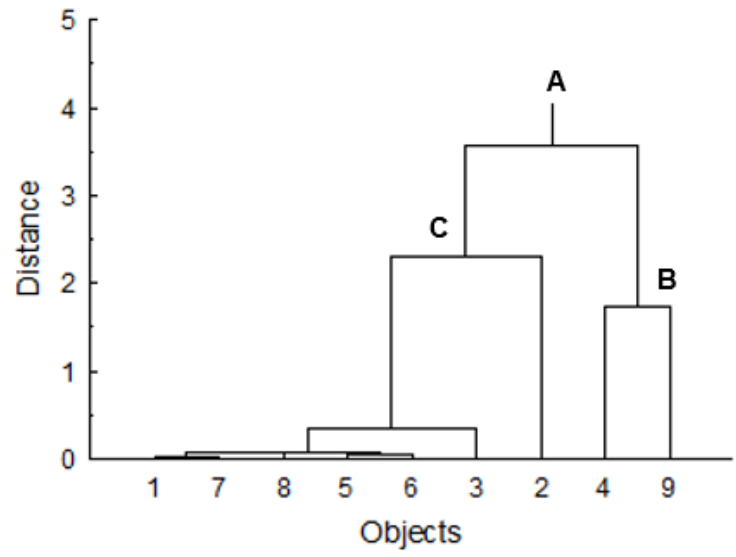

Figure 2 Vertical dendrogram of molecular properties (see Table 1), utilizing single linkage and Euclidean distance. Beginning with super node A, the compounds are distinguished into node $\mathrm{B}$ having compounds 4 and 9 (isoniazid). Compounds $1,7,8,5$, and 6 are seen to be most similar to each other, with compound 3 determined to be distinct. Compound 2 under node $\mathrm{C}$, is determined to be distinct from compounds $1,7,8,5,6$, and 3 .

The corresponding values of MIC (compound concentration when only $50 \%$ of the bacteria in culture are viable) for these hydrazide compounds are presented for comparison to isoniazid and for evaluation based on molecular structure (see Figure 3). The numerical values of MIC for hydrazide compounds 1, 2, 3, and 4 are essentially the same at 35 micrograms/milliliter. Interestingly, the substituents for compounds $1,2,3$, and 4, are diverse, having (see Figure 1): Compound 1) methyl group (meta); Compound 2) acetate group (meta); Compound 3) chloromethyl (para); Compound 4) nitro group (para). Notably, the substituents are in meta or para position to the hydrazide group, thus avoiding any steric hindrance to the action of the hydrazide moiety ($\left.\mathrm{C}(=\mathrm{O}) \mathrm{NHNH}_{2}\right)$. Compound 5 has the largest numerical value of MIC, requiring the greatest concentration of compound to achieve $50 \%$ kill rate of bacteria in culture. The bromine atom (-Br) is in the ortho position relative to the hydrazide group, potentially influencing bioactivity of the compound due to steric hindrance. Compounds 6, 7, and 8 have the lowest numerical values of MIC and very close to that for isoniazid (14.7 micrograms/milliliter). Both compounds 6 and 7 have bromine atoms as substituents, as does compound 5, however the bromine atoms are in meta position (compound 6) and para position (compound 7) relative to the hydrazide group with no steric hindrance as a potential hurdle to bioactivity.

Compound 8 is unique; it is a hydrazide derivative of ciprofloxacin $[\mathbf{1 5}, \mathbf{1 6}]$. Ciprofloxacin is a broadspectrum antibiotic of the fluoroquinolone class active against both Gram-positive and Gram-negative bacteria. Ciprofloxacin inhibits DNA gyrase, and topoisomerase IV, necessary to separate bacterial DNA [24]. Considering that the spectrum of activity includes most strains of bacterial pathogens responsible for respiratory, urinary tract, gastrointestinal, and abdominal infections [24], it follows that the bioavailability of ciprofloxacin is substantially favorable. This hydrazide derivative (compound 8) has numerical values of Log BB and Log P very close to that of isoniazid ( $\log \mathrm{P}$ for both are less than zero), these facts may provide some clue as to the similarity in MIC values. 


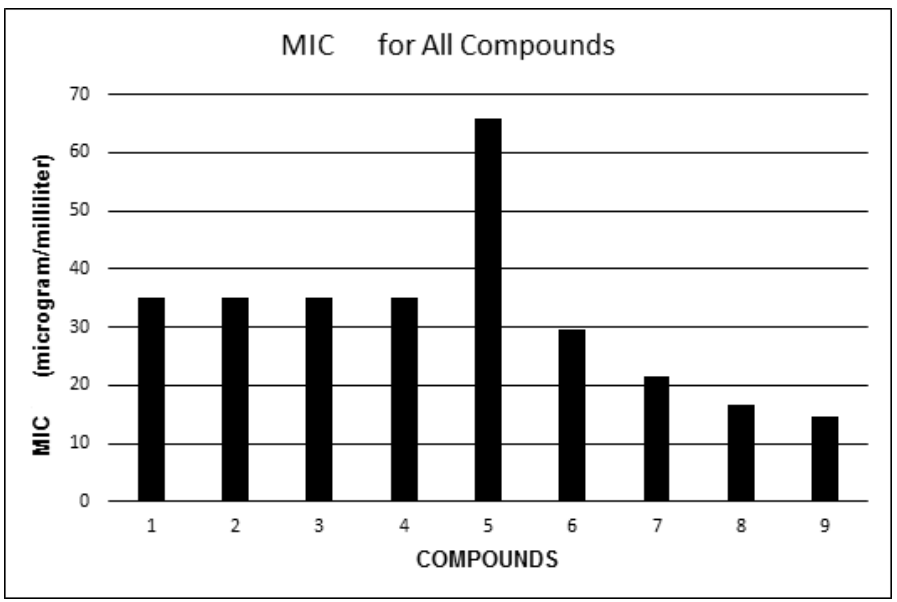

Figure 3 Values of MIC for all hydrazide compounds 1 to 8 . Compound 9 is isoniazid.

Path analysis is a straightforward extension of multiple regression. Two or more specified causal hypotheses can be represented in which the relative sizes of path coefficients in the output path relates which of them is better supported by the data $[\mathbf{2 5}, \mathbf{2 6}, \mathbf{2 7}]$. The larger the numerical value of the path coefficient, the greater the support for the caused variable (MIC in this study).

Results of path analysis for causal relationship of molecular properties for minimum inhibitory concentration (MIC) is presented in Table 3. The largest coefficients indicate the property determined to be of highest causal relationship of MIC of this tuberculostats. Clearly, the number of oxygen and nitrogen atoms have the greatest causal relationship to MIC having path coefficient of 5.578. This is followed second by Log P property, having a path coefficient of 1.490. This study elucidates the properties most important for achievement of favorable (lower) values of MIC and will assist in further drug development of hydrazide compounds for treatment of tuberculosis.

Table 3 Path Analysis for Summary Causal Relationship of Properties to MIC

\begin{tabular}{|l|l|l|}
\hline $\begin{array}{l}\text { Variable Caused } \\
\text { (Minimum inhibitory concentration) }\end{array}$ & Causing Variable & Path Coefficient \\
\hline MIC & Log P & 1.490 \\
\hline MIC & $\begin{array}{l}\text { Polar Surface Area } \\
\left(\text { Angstroms }{ }^{2}\right)\end{array}$ & -3.832 \\
\hline MIC & Number of Atoms & -2.000 \\
\hline MIC & Molecular Weight & -0.181 \\
\hline MIC & $\begin{array}{l}\text { Number of Oxygen } \\
\text { \& Nitrogen Atoms }\end{array}$ & 5.578 \\
\hline MIC & Number of Rotatable Bonds & 0.257 \\
\hline MIC & $\begin{array}{l}\text { Molecular Volume } \\
(\text { Angstroms }\end{array}$ & 0.630 \\
\hline
\end{tabular}

\section{Multiple Regression Analysis for Prediction}

Multiple regression is a statistical tool that allows you to examine how multiple independent variables are related to a dependent variable. Once you have identified how these multiple variables relate to your dependent variable, you can take information about all of the independent variables and use it to make much more powerful and accurate predictions concerning these variables [22, 23]. For equation $\{2\}$, MW is molecular weight, PSA is polar surface area, nAtoms is number of atoms, nON is number of oxygen \& nitrogen atoms, nOHNH is number of hydroxyl \& amine groups, nRotB is number of rotatable bonds, and volume is molecular volume.

$$
\begin{gathered}
\mathrm{MW}=-3023.0+10.243(\mathrm{PSA})-686.97(\mathrm{nAtoms})+186.22(\mathrm{nON})+840.35(\mathrm{nOHNH})+ \\
44.217(\mathrm{nRotB})+49.144(\text { volume })
\end{gathered}
$$

Value of $\mathrm{R}^{2}$ is 1.00 , indicating that the model explains $100 \%$ of the variance within the model. The $\mathrm{P}$ values for each variable and constant are $(P<.0001)$, therefore the model indicates that the constant $(-3023.0)$ and all independent variables contribute significantly to this model [23]. The study and design of new drugs to combat the appearance of drug resistant tuberculosis is a vital undertaking to maintain effective clinical treatment of this bacterial pathogen. 


\section{Conclusion}

The study of novel drugs for the treatment of tuberculosis is necessary due to the appearance of the multidrug-resistant and extensively drug-resistant tuberculosis. This study presents eight hydrazide compounds shown to inhibit growth of Mycobacterium tuberculosis and compares their molecular properties and potency to the first-line drug isoniazid. The minimum inhibitory concentration (MIC) for reduction of bacteria viability to $50 \%$ of origin ranged from 16.7 micrograms/milliliter to 35.0 micrograms/milliliter for compounds 1 to 8 . Although the molecular properties of all nine hydrazide compounds (including isoniazid) varied in numerical values, the Pearson $r$ correlation of compound to other compounds showed a very strong positive relationship $r$ $>0.9700$. All nine compound showed zero violations of the Rule of 5. Compounds 1, 3, 5, 6, and 7 have greater penetration into the CNS, having values of BB greater than that of Isoniazid at 0.106. Hierarchical cluster analysis determined that compound 4 is most similar to isoniazid. Multiple regression analysis determined a mathematical model explaining $100 \%$ of variance in the variables and capable of predicting descriptor values for similar agents. Path analysis determined that number of oxygen and nitrogen atoms (path coefficient $=5.578$ ) and $\log \mathrm{P}$ (path coefficient $=1.490$ ) have highest causal relationship to values of MIC. The design and development of new tuberculostats are of vital importance for effective treatment of the spreading incidents of drug resistant Mycobacterium tuberculosis.

\section{Acknowledgements}

This study was supported by the College of Arts \& Sciences, Durham Science Center University of Nebraska, Omaha Nebraska 68182 USA; SDG.

\section{References}

[1]. F Drobniewski, Diagnosing multidrug resistant tuberculosis in Britain, BMJ, 317(7166), 1996, $1263-64$.

[2]. F Kurniawati, SA Syed Sulaiman, and SW Gillani, Study on drug-resistant tuberculosis and tuberculosis treatment on patients with drug resistant tuberculosis in chest clinic outpatient department. Int J Pharm Pharm Sci, 4(2), 2012, 733-37.

[3]. A Fauci, Multidrug-resistant and extensively drug-resistant tuberculosis: the national institute of allergy and infectious diseases research agenda and recommendations for priority research, J Infect Dis, 197(11), 2008, 1493-98.

[4]. J van den Boogaard, G Kibiki, E Kisanga, M Boecree, and R Aarnoutse, New drugs against tuberculosis: problems, progress, and evaluation of agents in clinical development, Antimicrob Agents Chemother, 53(3), 2009, 849-62.

[5]. P Davies, Drug-resistant tuberculosis, J R Soc Med, 94(6), 2001, 261-63.

[6]. M Iseman, Treatment of multi-drug resistant tuberculosis, N Engl J Med, 329, 1993, 784-91.

[7]. A Mwinga, Africa, in Davies PDO (Ed), Clinical Tuberculosis, $2^{\text {nd }}$ ed. (London, England: Chapman \& Hall, 1998).

[8]. M Berry, and O Kon, Multidrug- and extensively drug-resistant tuberculosis: an emerging threat, Eur Respr Rev, 18(114), 2009, 195-97.

[9]. N Shah, A Wright, G Bai, L Barrera, F Boulahbal, N Martan-Casabona, et al, Worldwide emergence of extensively drug-resistant tuberculosis, Emerg Infect Dis, 13, 2007, 380-7.

[10]. Tuberculosis (TB), Centers for Disease Control and Prevention Centers for Disease Control and Prevention, How TB Spreads, Accessed 15 December 2016. Available: https://www.cdc.gov/tb/topic/basics/howtbspreads.htm.

[11]. Center for Disease Control, Emergence of Mycobacterium tuberculosis with extensive resistance to second-line drug-worldwide, MMWR Morb Mortal Wkly Rep, 55(11), 2006, 301-5.

[12]. D Kumar, B Negi, and D Rawat, The anti-tuberculosis agents under development and the challenges ahead, Future Med Chem, 7(15), 2015, 1981-2003.

[13]. M Haas, and R Belknap, A review of rifapentine for the treating active and latent tuberculosis, Clinical Investigation, 5(11), 2015, 829-38.

[14]. J van den Boogaard, G Kibiki, E Kisanga, M Bocree, and R Aarnoutse, New drugs against tuberculosis: problems, progress, and evaluation of agents in clinical development, Antimicrob Agents Chemother, 53(3), 2009, 849-62.

[15]. R Bartzatt, P Sule, W Kim, and J Cirillo, Four compounds suppressing growth of Mycobacterium tuberculosis, JAMPS, 6(4), 2016, $1-10$.

[16]. R Bartzatt, SLG Cirillo, and J Cirillo, Hydrazide drugs that inhibit growth and proliferation of tuberculosis bacteria, Physiol Chem Phys \& Med NMR, 41, 2011, 49-59.

[17]. C Lipinski, F Lombardo, B Dominy, and P Feeney P, Experimental and computational approaches to estimate solubility, and permeability in drug discovery and development settings, Adv Drug Deliv Rev, 46(1-3), 2001, 3-26.

[18]. C Lipinski, Lead- and drug-like compounds: the rule-of-five revolution, Drug Discov Today Technol, 1(4), $2004,337-41$.

[19]. D Veber, S Johnson, H Cheng, B Smith, K Ward, and K Kopple, Molecular properties that influence the oral bioavailability of drug candidates, J Med Chem, 45(12), 2002, 2615-23.

[20]. OA Raevsky, Physiochemical descriptors in property-based drug design, Mini-reviews Med Chem, 4, $2004,1041-52$.

[21]. D Clark, Rapid calculation of polar molecular surface area and its application to the prediction of transport phenomena. 2. Prediction of blood-brain barrier penetration, J Pharm Sci, 88(8), 1999, 815-21.

[22]. R Sokal, and J Rohlf, Biometry (San Francisco, CA: W.H. Freeman, 1995).

[23]. O Hammer, and D Harper, Paleontological Data Analysis (New York, NY: Blackwell, 2006).

[24]. K Drlica, and X Zhao, DNA gyrase, topoisomerase IV, and the 4-quinolones, Microbiology and Molecular Biology Reviews, 61(3), 1997, 377-92.

[25]. S Wright, The method of path coefficients, Annals of Mathematical Statistics, 5(3), 1934, 161-215.

[26]. DR Heise, Causal analysis (New York, NY: John Wiley \& Sons, 1975).

[27]. RP McDonald, Path analysis with composite variables. Multivariate Behavioral Research, 31(2), 1996, $239-270$. 